\title{
Pancreatic Glucagonoma
}

National Cancer Institute

\section{Source}

National Cancer Institute. Pancreatic Glucagonoma. NCI Thesaurus. Code C95597.

A usually malignant, glucagon-producing neuroendocrine tumor arising from the pancreatic alpha cells. It may be associated with necrolytic erythema migrans, diarrhea, diabetes, glossitis, weight loss, malabsorption, and anemia. Almost $25 \%$ of patients experience thromboembolic complications, and 50\% have liver metastases at the time of diagnosis. 